\title{
Reconfigurable Microstrip Patch Antenna using MEMS Technology
}

\author{
E.Ramola ${ }^{1}$, Dr.T.Pearson ${ }^{2}$ \\ ${ }^{I}$ (ECE, D.M.I College of Engineering/ Anna University, India) \\ ${ }^{2}$ (Dean-ECE, D.M.I College of Engineering/ Anna University, India)
}

\begin{abstract}
This project proposes the design of a Reconfigurable Micro Strip Patch Antenna Using MEMS Technology A Reconfigurable Microstrip patch antenna of operating frequency in the range of 5-8 $\mathrm{GHz}(\mathrm{C}$ Band), for the application of wireless communication has been designed. The term Reconfigurability refers that it adaptively changes its characteristics. A MEMS-based switch is inserted in the patch to control its configuration. Patch antenna using switchable slots shows different resonant features with different states (ON $\& O F F)$ of the switch. When the switch is in the OFF mode, the electric currents on the patch have to flow around the slot, resulting in a relatively long length of the current path. Therefore, the antenna resonates at a low frequency. In contrast, when the switch is in the ON mode, some of the electric currents can go directly through the switch. In this case, the average length of the current path is shorter, so that the antenna has a higher resonant frequency. The antenna was designed and analyzed using ANSOFT HFSS.
\end{abstract}

Keywords - Reconfigurable Antenna, Frequency Diversity, Patch Antenna, Switchable Slot

\section{INTRODUCTION}

In the field of wireless communications, a very important development is the integration of different applications including cellular phones, satellite communications and wireless local area networks. Each of these applications operates on a different frequency band; each of these circuits requires different configurations. Due to size and hence the complexity \& cost constraints in designing such devices, it would be beneficial to have reconfigurable circuitry that operates on each of these different frequencies. A Reconfigurable microstrip patch antenna is an antenna which can expand the system capability by adjusting its resonant frequency, radiation pattern and polarization. Reconfigurable antennas are becoming more important in defense and commercial wireless applications, since with such antennas, a single aperture can be used to support multiple functions at multiple frequency bands. This will result in a significant reduction in antenna size and cost.

The patch antenna [1] with Right Hand Circular Polarization (RHCP) and Left Hand Circular Polarization (LHCP) with a single feeding port designed by Mohammod Ali, describes about two orthogonal slots are incorporated into the patch and two P I N diodes are utilized to switch the slots ON and OFF. The unique feature of this concept is RHCP \&LHCP are time separated, (depending on the switch time of the diodes) so that there is no coupling between these two polarizations. The switching speed of the PIN diodes is slower than the MEMS-based switches. Nanbo Jin designed the patch antenna [2] with switchable slot (PASS).This concept is implemented to design a novel reconfigurable antenna with both frequency and polarization diversities. Using only one switch and a single patch, the antenna operates at $4.20 \mathrm{GHz}$ and at $4.55 \mathrm{GHz}$.The fabricated antenna has both an acceptable return loss and broadside axial ratio lower than $2 \mathrm{db}$ at each operation frequencies and polarization diversity. Single schottky diode is used to achieve dual frequency and polarization diversities. The schottky diode has the insertion loss of $2 \mathrm{db}$ and operates at low speed. Using PIN diode switches, Rahmat Samii designed a stacked micro strip antenna [3] that can be reconfigured to support two different frequency bands with varying bandwidth pattern and polarization requirements. In this paper the stacked design is operating at $1.75 \mathrm{GHz}$. The antenna is used in the lower band terrestrial and land-mobile applications.

In this paper, the Reconfigurable Microstrip Patch Antenna using Micro Electro Mechanical Systems (MEMS) technology for micrometer wave application was designed. The reconfigurable antenna is the antenna which adaptively changes it characteristics. The Reconfigurability is done by changing the status (ON \& OFF) of the MEMS cantilever switch. MEMS technology is a hot technology, to integrate both microelectronic circuits and mechanical structures on the same chip, enabling monolithic integration while reducing the micro system size and cost considerably. The critical physical dimensions of MEMS devices can vary from well below one micron to several millimeters. These switches have near-zero power consumption, very high isolation, very low insertion loss and very high switching speed. 


\subsection{Introduction}

\section{RECONFIGURABLE ANTENNA}

The gain-bandwidth limitation of electrically small antennas is a fundamental law of physics that limits the ability of the wireless-system engineer to reduce the footprint of the antenna while maintaining or increasing its efficiency. A revolutionary approach to circumventing that limitation is to construct a small, highly efficient antenna with a narrow instantaneous operating bandwidth that can be tuned over a much wider operating bandwidth. The concept was first implemented for small, low-profile antennas that provide adequate gain over the UHF Satcom band (240 to $320 \mathrm{MHz}$ ) in high-performance military aircraft, where weight and size are significant design constraints. The antenna employed solid-state switch elements, namely PIN diodes, to dynamically adjust the effective electrical size and, hence, the operating frequency of a microstrip patch antenna over a large frequency band while maintaining an excellent impedance match and efficiency. Although the approach appear to increase the complexity and expense relative to a conventional passive antenna, a more thorough analysis reveals advantages in cost and system architecture design for current $3 \mathrm{G}$ systems. Indeed, we have found that selectivity of the reconfigurable antenna provides filter-like performance so that duplexing, diplexing and band pass image rejection may be absorbed into the antenna. That simplifies the transceiver architecture, reducing cost, enhancing quality of service and reducing requirements on prime power.

Simple Microstrip antennas radiate linearly polarized fields over a 1-2\% bandwidth. By modifying the geometry of the antenna or using a dual feed network with phase shift it is possible to radiate circularly polarized radiation. Unfortunately, these changes are permanent and they cannot be used to reconfigure the antennas performance. Electronic control using varactor diodes as oscillators or variable transmission line lengths has been demonstrated. It was also demonstrated that by changing the number and location of shorting positions, both the operating frequency and polarization of the patch could be controlled. All these techniques require a continuous and precise supply of DC bias and accurate positioning of diodes on the patch surface or addition of phase shifting networks near the patch antenna; all of which perturbs the radiated field shape and polarization purity. In this paper these disadvantages are overcome by using RF MEMS switches integrated into the patch antenna's ground plane and connected with shorting via to the upper plate. Compared with pin or varactor diodes, MEMS consumes little DC power and have low insertion loss and high isolation. A GPS polarization reconfigurable patch antenna has been experimentally investigated using RF MEMS switches. A square patch antenna designed with four pairs of permanent shorting via for obtaining four different polarizations. The design is then similar to one with removable shorting posts. Here, the effects of MEMS integrations and parasitic bond wires on antenna performance are quantified and compared with an identical patch antenna using removable shorting posts. In this project we show how to increase the number of resonant frequency and bandwidth of the previously developed PASS antenna using stacked micro strip configuration concept [2]. The reconfigurable micro strip patch antenna designed using RF MEMS switches resonates at two different frequencies with switch ON and OFF modes.

\subsection{Basic Principle of Operation}

The metallic patch essentially creates a resonant cavity, where the patch is the top of the cavity, the ground plane is the bottom of the cavity, and the edges of the patch form the sides of the cavity. The edges of the patch act approximately as an open-circuit boundary condition. Hence, the patch acts approximately as a cavity with perfect electric conductor on the top and bottom surfaces, and a perfect magnetic conductor on the sides. This point of view is very useful in analyzing the patch antenna, as well as in understanding its behavior. Inside the patch cavity the electric field is essentially $\mathrm{z}$ directed and independent of the $\mathrm{z}$ coordinate. Hence, the patch cavity modes are described by a double index $(m, n)$. For the $(m, n)$ cavity mode of the rectangular patch the electric field has the form:

$$
\mathrm{E}_{\mathrm{z}}(\mathrm{x}, \mathrm{y})=\mathrm{A}_{\mathrm{mn}} \cos (\mathrm{m} \pi \mathrm{x} / \mathrm{L}) \cos (\mathrm{n} \pi \mathrm{y} / \mathrm{W}) \quad-----(1)
$$

The patch is usually resonates at $(1,0)$ mode and the above field in (1) is essentially constant in the $y$ direction. The surface current on the bottom of the metal patch is then $\mathrm{x}$ directed, and is given by:

$$
\mathrm{J}_{\mathrm{zx}}(\mathrm{x})=\mathrm{A}_{10}\left((\pi / \mathrm{L}) / \mathrm{j} \omega \mu_{0} \mu_{\mathrm{r}}\right)
$$

For this mode the patch may be regarded as a wide microstrip line of width $\mathrm{W}$, having a resonant length $\mathrm{L}$ that is approximately one-half wavelength in the dielectric. The current is maximum at the centre of the patch, $\mathrm{x}=\mathrm{L} / 2$, while the electric field is maximum at the two "radiating" edges, $\mathrm{x}=0$ and $\mathrm{x}=\mathrm{L}$. The width $\mathrm{W}$ is usually chosen to be larger than the length ( $\mathrm{W}=1.5 \mathrm{~L}$ is typical) to maximize the bandwidth, since the bandwidth is proportional to the width. 


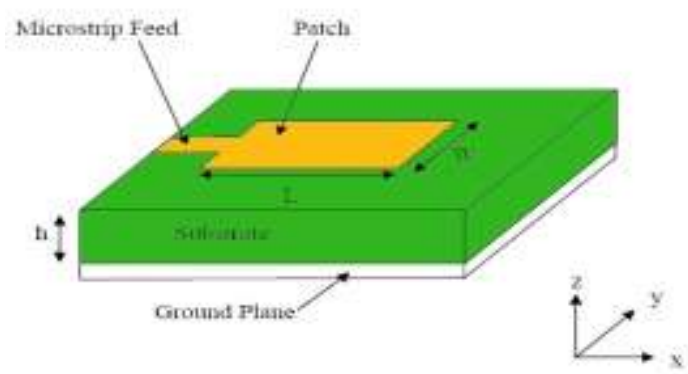

Fig 1: Microstrip Patch Antenna

At first glance, it might appear that the microstrip antenna will not be an effective radiator when the substrate is electrically thin, since the patch current in (2) will be effectively shorted by the close proximity to the ground plane. If the modal amplitude $\mathrm{A}_{10}$ is a constant, the strength of the radiated field would be proportional to $\mathrm{h}$. However, the $\mathrm{Q}$ of the cavity increases as $\mathrm{h}$ decreases. Hence, the amplitude $\mathrm{A}_{10}$ of the modal field at resonance is inversely proportional to $h$. Hence, the strength of the radiated field from a resonant patch is essentially independent of $\mathrm{h}$, if losses are ignored. The resonant input resistance will likewise be nearly independent of $\mathrm{h}$. This explains why a patch antenna can be an effective radiator even for very thin substrates, although the bandwidth will be small.

\subsection{RF-MEMS Switch}

Micro Electronic Mechanical System (MEMS) based technology normally includes a substrate with one or more metal traces and control pads. MEMS switch may be constructed which uses electrostatic force to flex a thin membrane and thereby make the switch to be opened or closed. Since an electrostatic force is used, the switch can be controlled using only a voltage and consumes virtually no power. This is an important advantage for portable communication systems, such as hand-held mobile phones or other wireless communication devices, where power consumption is recognized as a significant operating limitation.

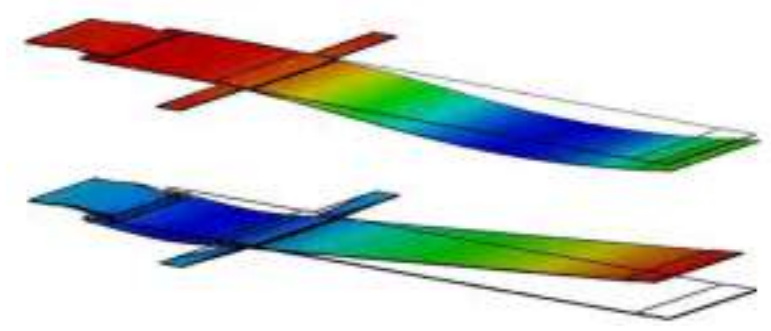

Fig 2: RF-MEMS Cantilever Type Switch

In this paper we are using a capacitive type MEMS switch in which a dielectric layer is deposited on the first conductor in an area opposite to the underside of the two-arm moveable bridge, with this area on the conductor acting as the pull down electrode. With this arrangement, the full pull down voltage appears across the dielectric layer resulting in a relatively high electric field across the dielectric. Radio frequency (RF) MEMS switches with very high switching speed are commonly used in communication systems, where high frequency operation is required. A radio frequency (RF) switch is a device that controls the flow of an RF signal. MEMS switches are termed electrostatic MEMS switches if they are actuated or controlled using electrostatic force which turns such switches on and off. Electrostatic MEMS switches are advantageous due to low powerconsumption because they can be actuated using electrostatic force induced by the application of a voltage with virtually no current. The MEMS devices offer the following advantages compared to semiconductor devices. First the significant reduction in insertion loss resulted in higher figure of merit. Second, they consume insignificant amount of power during operation which results in higher efficiency. Third, they exhibit higher linearity and as a result lower signal distortion when compared to semiconductor devices. However, the implementation of RF-MEMS switches does not come with impurity.

\section{Antenna Design}

The Reconfigurable micro strip patch antenna for wireless application operating in C-frequency band was designed and the parameters were simulated using ANSOFT High Frequency Structural Simulator (HFSS). 


\subsection{Design Specifications}

The three essential parameters for the design of the Rectangular Microstrip Patch Antenna:

\subsubsection{Resonant Frequency $\left(\mathbf{f}_{\mathbf{0}}\right)$}

The resonant frequency of the antenna must be selected appropriately. It is given by the equation: $\mathrm{f}_{0}=\mathrm{c} / 2 \mathrm{Le} \sqrt{ } \varepsilon_{\mathrm{r}}$

The wireless communication system operating in the frequency range of $\mathrm{C}$ - band is selected. Hence the antenna designed must be able to operate in this frequency range. The resonant frequency selected for my design is 6 GHz. The antenna designed will adaptively change its characteristic with respect to the position of switch. The antenna resonates at 5.38, 5.68, 5.75, $6 \mathrm{GHz}$.

\subsubsection{Dielectric constant of the substrate $(\square$ )}

The dielectric material selected for our design is RT Duroid which has a dielectric constant of 2.20009.A substrate with a high dielectric constant has been selected since it reduces the dimensions of the antenna.

\subsubsection{Height of dielectric substrate (h)}

For the microstrip patch antenna the substrate thickness impact on Bandwidth. For the C-Band operation the antenna should not be a thick. So the height of the dielectric substrate is selected as $1 \mathrm{~mm}$.

\section{Design Parameters}

To design the patch antenna of required frequency we have to calculate the following parameters:

\subsection{Calculation of Width}

The width of the Microstrip patch antenna is given as:

$$
W=\frac{c}{2 f_{\circ} \sqrt{\frac{\left(\varepsilon_{r}+1\right)}{2}}}
$$

Substituting $\mathrm{c}=3.00 \mathrm{e}+008 \mathrm{~m} / \mathrm{s}, \varepsilon_{\mathrm{r}}=2.2$ and $\mathrm{f}_{0}=6 \mathrm{GHz}$, we get, $\mathrm{W}=0.0197642 \mathrm{~m}$ (or) $\mathrm{W}=19.7642 \mathrm{~mm}$.

\subsection{Calculation of Effective dielectric constant ( $\square$ reff)}

The effective dielectric constant is:

$\varepsilon_{\text {reff }}=\left(\varepsilon_{\mathrm{r}}+1 / \varepsilon_{\mathrm{r}}\right)+\left(\varepsilon_{\mathrm{r}}-1 / \varepsilon_{\mathrm{r}}\right) \sqrt{ }(1+12 \mathrm{~h} / \mathrm{W})$

Substituting $\varepsilon_{\mathrm{r}}=2.2, \mathrm{~W}=19.7642 \mathrm{~mm}$ and $\mathrm{h}=1 \mathrm{~mm}$ we get, $\quad \varepsilon_{\text {reff }}=5.5032$

\subsection{Calculation of the length $(L)$}

The length is given by:

$\mathrm{L}=0.49 \lambda / \square \varepsilon_{\mathrm{r}} \quad$-.----- (6)

Substituting $\varepsilon_{\mathrm{r}}=2.2, \mathrm{c}=3.00 \mathrm{e}+008 \mathrm{~m} / \mathrm{s}$ and $\mathrm{f}_{0}=6 \mathrm{GHz}$ we get, $\mathrm{L}=.0165178 \mathrm{~m}$ (or) $\mathrm{L}=16.5178 \mathrm{~mm}$

\section{Designed Antennas}

The frequency shift can be explained by investigating the electric currents on the patch antennas. When the switch is in the OFF mode, the electric currents on the patch have to flow around the slot, resulting in a relatively long length of the current path. Therefore, the antenna resonates at a low frequency. In contrast, when the switch is in the ON mode, some of the electric currents can go directly through the switch. In this case, the average length of the current path is shorter, so that the antenna has a higher resonant frequency. PASS show different resonant features with different states of the switch.

\subsection{Antenna without Switch}

The MSPA was designed to operate in C-Band that resonates at 5.38GHz. For switching cantilever type switch is used. The switch is removed from simulation when the switch is in OFF state.

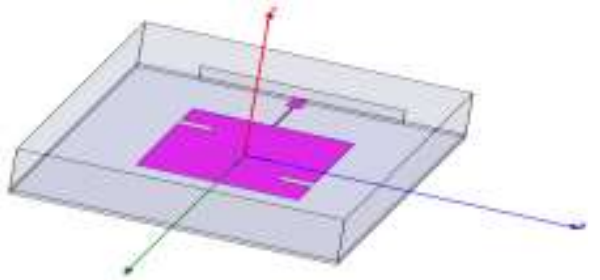

FIG 3: MSPA WITHOUT SWITCH 


\subsection{Antenna with First Switch On}

The MSPA was designed to operate in C-Band that resonates at $5.725 \mathrm{GHz}$. The first switch is in ON mode it is added in the simulation. The second switch is in OFF mode so it is removed from simulation.

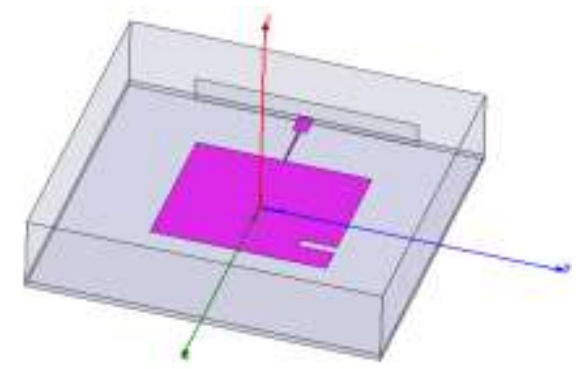

Fig 4: MSPA with First Switch ON

\subsection{Antenna With Second Switch On}

The MSPA was designed to operate in C-Band that resonate at $5.68 \mathrm{GHz}$. The first switch is in OFF mode it is removed from the simulation. The second switch is in ON mode so it is added to the simulation.

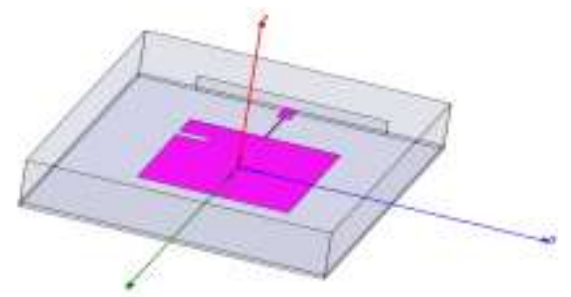

Fig 5: MSPA with Second Switch ON

\subsection{Antenna with Both Switch On}

The MSPA was designed to operate in C-Band that resonates at 6GHz. Both the switch is in ON mode and it is added in the simulation.

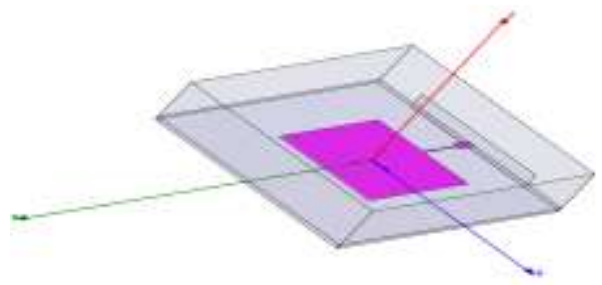

Fig 6: MSPA with Both Switches ON

\subsection{RETURN LOSS}

\section{Results And Discussion}

The micro strip line feed is used in the design the MSPA. Feed-line width of $1.5564 \mathrm{~mm}$ and Feed-line length of $2.4563 \mathrm{~mm}$ had been taken. A frequency range of 5-6 GHz is selected. The center frequency is selected as the one at which the return loss is minimum. The bandwidth of the antenna can be said to be those range of frequencies over which the Return Loss (RL) is greater than $-12 \mathrm{~dB}(-12 \mathrm{~dB}$ corresponds to a VSWR of 1.5 which is an acceptable figure). A RL of $-30 \mathrm{~dB}$ is obtained.

\subsection{VSWR PLOTS}

When a transmitter is connected to an antenna by a feed line, the impedance of the antenna and feed line must match exactly for maximum energy transfer from the feed line to the antenna to be possible. When an antenna and feed line do not have matching impedances, some of the electrical energy cannot be transferred from the feed line to the antenna. Energy which is not transferred to the antenna is reflected back towards the transmitter. The parameter VSWR is a measure that numerically describes how well the antenna is impedance matched to the radio or transmission line it is connected to. VSWR stands for Voltage Standing Wave Ratio, and it is the function of the reflection coefficient, which describes the power reflected from the antenna. In this paper, VSWR lie in the range of 1-2 which is achieved for all the four frequencies (5\% returns). 


\subsection{Radiation Pattern Plots}

Microstrip patch antenna radiates normal to its patch surface, the elevation pattern for $\varphi=0$ and $\varphi=90$ degrees would be important to obtain the gain of the antenna at 5.38, 5.68, 5.725\& $6 \mathrm{GHz}$. The maximum gain is obtained in the broadside direction and this is measured to be $7.2-8 \mathrm{dBi}$ for $\varphi=0$ and for $\varphi=90$ degrees.

\subsection{Mspa With Out Switches}

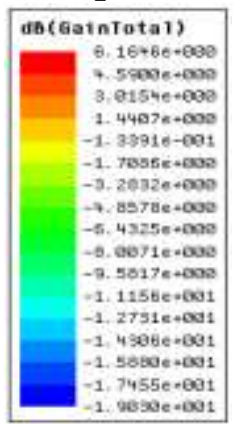

Fig 7: 3-D Radiation Pattern

\section{Simulated Results}

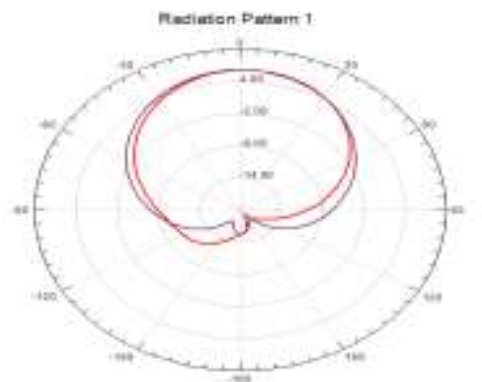

Fig 9: Radiation Pattern (Smith Chart)

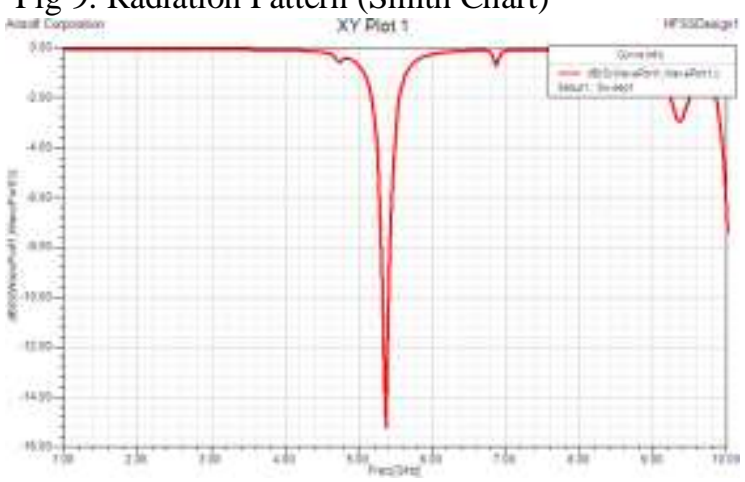

Fig 11: S-Parameter
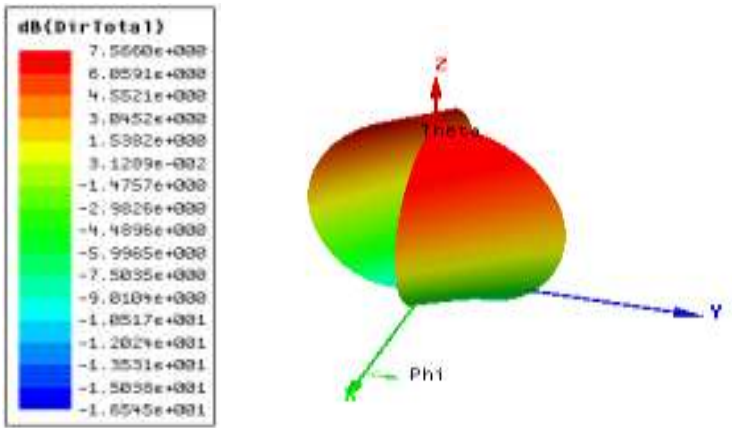

Fig 8: Directivity

\begin{tabular}{|l|c|}
\hline \multicolumn{1}{|c|}{ Curve Info } & xdb10Beamw idth(3) \\
\hline $\begin{array}{l}\text { Setup1 } 1 \text { : LastA daptive } \\
\text { Phi='Odeg' }\end{array}$ & 69.9600 \\
\hline $\begin{array}{l}\text { Setup1 : LastA daptive } \\
\text { Phi='90deg' }\end{array}$ & 76.9985 \\
\hline
\end{tabular}

Fig 10: Gain of MSPA with Out Switch

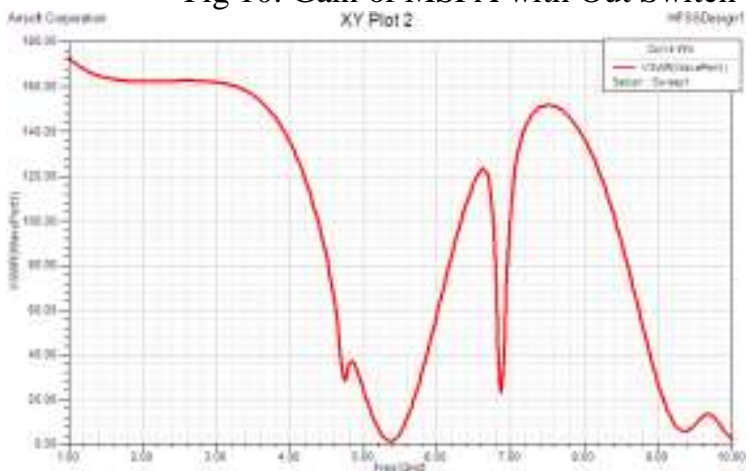

Fig 12: VSWR -Plot

\subsection{Mspa With First Switch On}

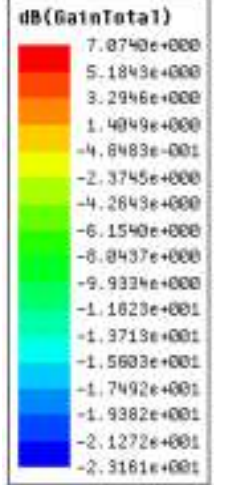

Fig 13: 3-D Radiation Pattern

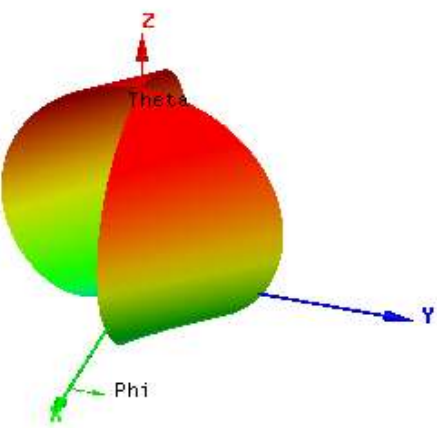

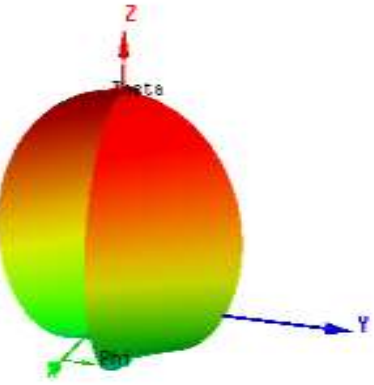




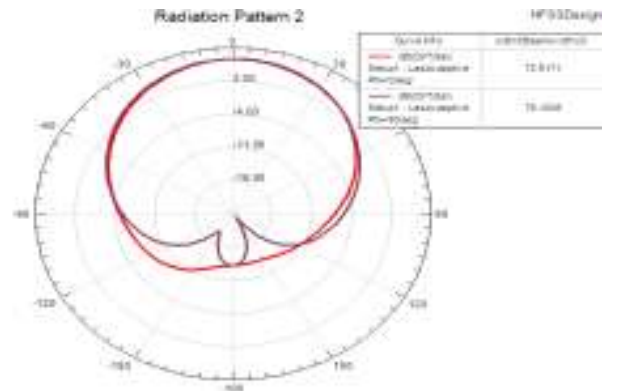

Fig 15: Radiation Pattern (Smith Chart)

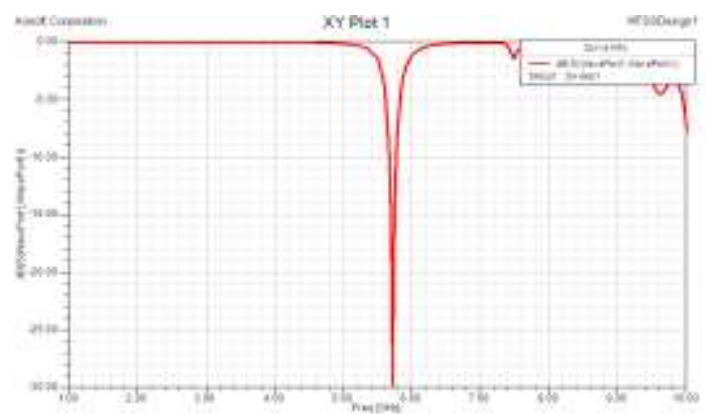

Fig17: S-Parameter

\begin{tabular}{|c|c|}
\hline Curve Info & xdb10Beamw idth(3) \\
\hline $\begin{array}{l}\text { dB(GainTotal) } \\
\text { Setup 1 : LastAdaptive } \\
\text { Phi='0deg' }\end{array}$ & 72.8755 \\
\hline $\begin{array}{l}\text { dB(GainTotal) } \\
\text { Setup1 : LastAdaptive } \\
\text { Phi='90deg' }\end{array}$ & 76.0911 \\
\hline
\end{tabular}

Fig16: Gain Of MSPA with First Switch ON

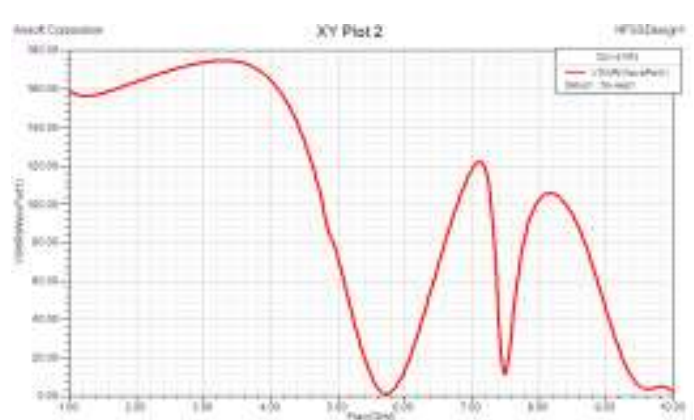

Fig18: VSWR Plot

\subsection{Mspa With Second Switch On}

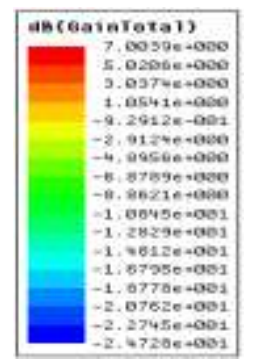

Fig 19: 3-D Radiation Pattern
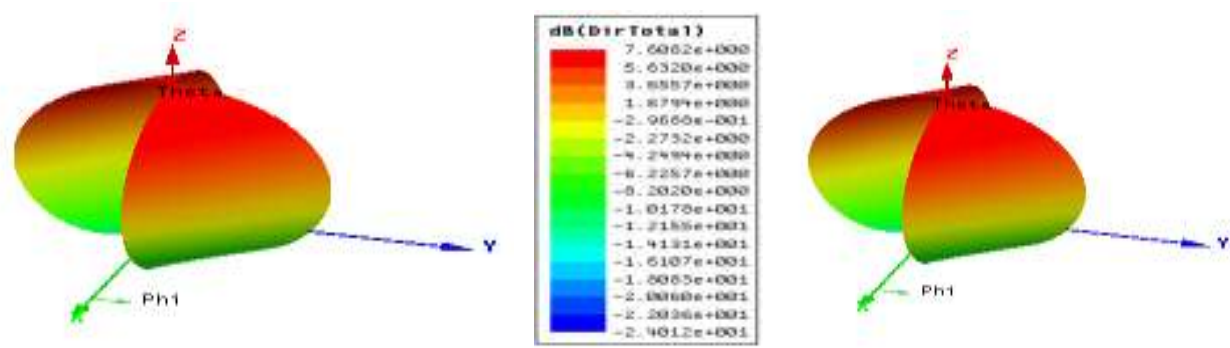

Fig 20: Directivity

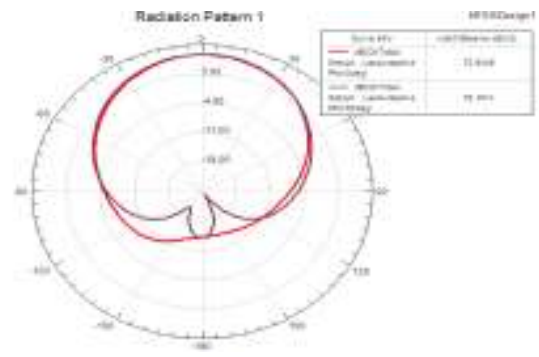

Fig 21: Radiation Pattern (Smith Chart)

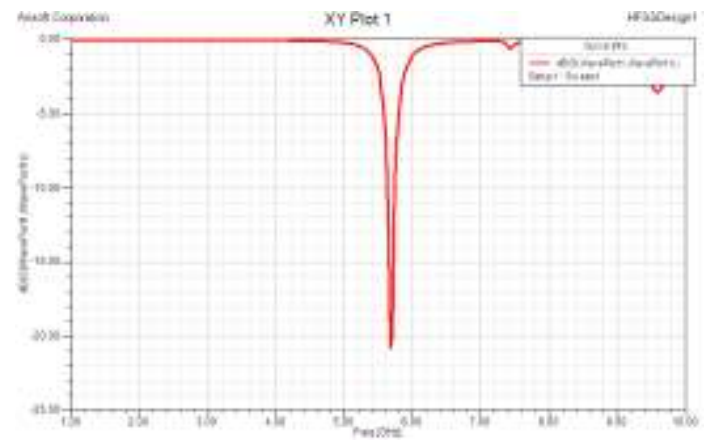

Fig 23: S-Parameter

\begin{tabular}{|l|c|}
\hline \multicolumn{1}{|c|}{ Curve Info } & xdb10Beamw idth(3) \\
\hline $\begin{array}{l}\text { Setup1 : LastA daptive } \\
\text { Phi='Odeg' }\end{array}$ & 72.9171 \\
\hline $\begin{array}{l}\text { Setup1 : LastAdaptive } \\
\text { Phi=90deg' }\end{array}$ & 76.1006 \\
\hline
\end{tabular}

Fig 22: Gain Of MSPA With Second Switch

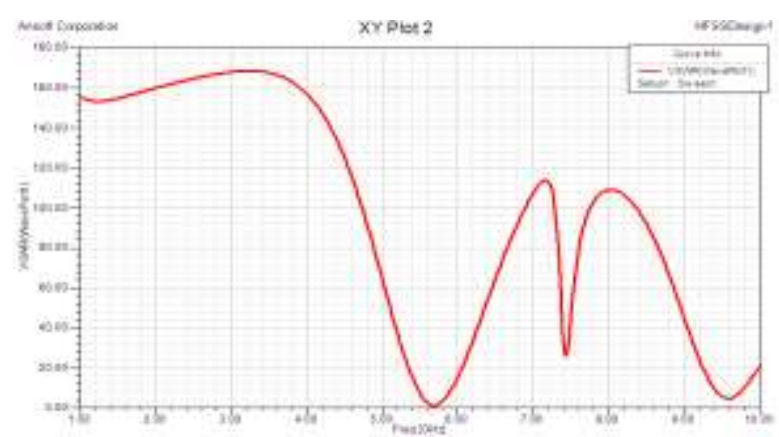

Fig24: VSWR Plot 


\subsection{Mspa With Both Switches On}

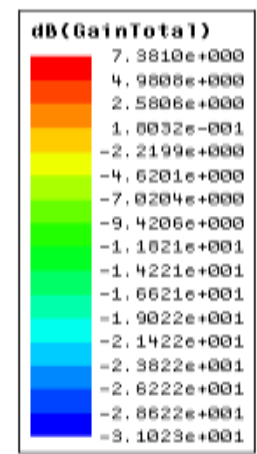

Fig 25: 3-D Radiation Pattern

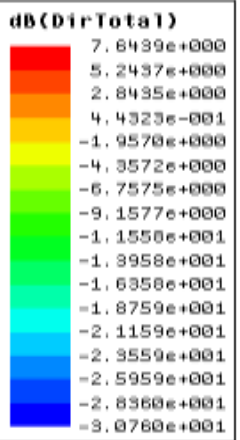

Fig 26: Directivity

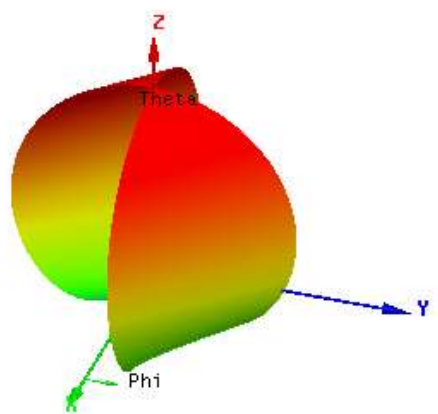

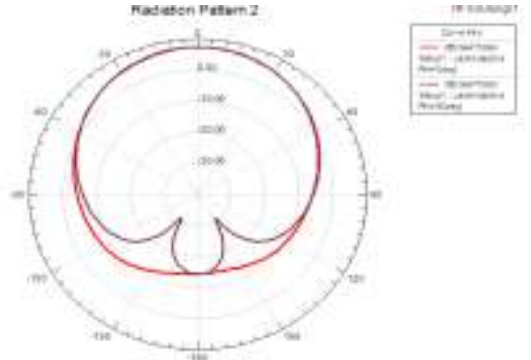

Fig 27: Radiation Pattern (Smith Chart)

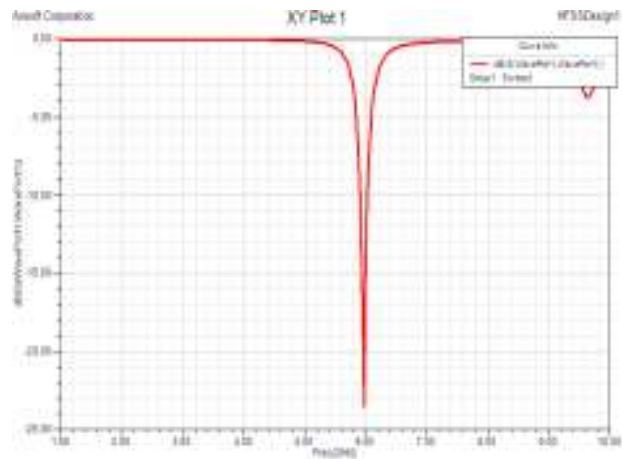

Fig 29: S-Parameter

\begin{tabular}{|l|c|}
\hline \multicolumn{1}{|c|}{ Curve Info } & xdb10Beamwidth(3) \\
\hline $\begin{array}{l}\text { Setup1 : LastA daptive } \\
\text { Phi='0deg' }\end{array}$ & 74.7796 \\
\hline $\begin{array}{l}\text { Setup1 : LastAdaptive } \\
\text { Phi=90deg' }\end{array}$ & 75.1774 \\
\hline
\end{tabular}

Fig28: Gain Of MSPA with Both Switch

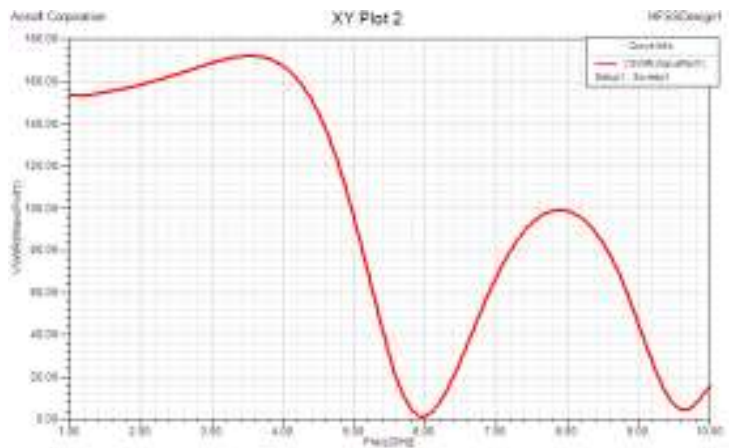

Fig 30: VSWR Plot

\section{CONCLUSION}

The design of a Reconfigurable Microstrip Patch Antenna with resonant frequency of $6 \mathrm{GHz}$ was designed using ANSOFT HFSS and the different operating frequencies of $5.38,5.68,5.75 \mathrm{GHz}$ we re obtained using RF MEMS switch. The Reconfigurable microstrip patch antenna can expand the system capability. By adjusting its resonant frequency, radiation pattern and polarization the reconfigurable antenna can accommodate various requirements of wireless communication. Microstrip antennas are widely used in satellite and mobile communications systems because of their light weight and planar shape. Any way we have to overcome the following disadvantages: extraneous radiation from feeds and junctions, poor end fire radiator except tapered slot antennas, low power handling capacity and surface wave excitation.

\section{REFERENCES}

[1] A Reconfigurable Stacked Microstrip Patch Antenna for Satellite and Terrestrial Links by Mohammod Ali, IEEE transactions on vehicular technology, vol. 56, no. 2, march 2007

[2] A Novel Patch Antenna with Switchable Slot (Pass): Dual-Frequency Operation with Reversed Circular Polarization by Nanbo Jin,Fan Yang and Yahya Rahmat-Samii , IEEE transactions on antennas and propagation, vol. 54, no. 3, march 2006

[3] A Reconfigurable Patch Antenna Using Switchable Slots for Circular Polarization Diversity by Rahmat Samii \& Yang, IEEE Microwave And Wireless Components Letters, Vol. 12, No. 3, March 2002

[4] A Wideband E-Shaped Microstrip Patch Antenna For 5-6 Ghz Wireless Communications by B.-K. Ang and B.-K. Chung ,Progress In Electromagnetics Research, PIER 75, 397-407, 2007 